\title{
Hyaluronic acid and tendon lesions
}

\author{
Jean-François Kaux ${ }^{1,2,3}$ \\ Antoine Samson ${ }^{1,2}$ \\ Jean-Michel Crielaard ${ }^{1,2,3}$ \\ 1 Department of Physical Medicine and Sports Trau- \\ matology, CHU Liège, Belgium \\ 2 Multidisciplinary Department for Medicine and Sports \\ Traumatology (SPORTS), CHU Liège, Belgium \\ 3 Department of Sports and Rehabilitation Sciences, \\ University of Liège, Belgium
}

Corresponding author:

Jean-François Kaux

Department of Physical Medicine and Sports Traumatology, $\mathrm{CHU}$ and University of Liège

Avenue de l'Hôpital, B35

4000 Liège, Belgium

E-mail: jfkaux@chu.ulg.ac.be

\section{Summary}

Introduction: recently, the viscoelastic properties of hyaluronic acid (HA) on liquid connective tissue have been proposed for the treatment of tendinopathies. Some fundamental studies show encouraging results on hyaluronic acid's ability to promote tendon gliding and reduce adhesion as well as to improve tendon architectural organisation. Some observations also support its use in a clinical setting to improve pain and function. This literature review analyses studies relating to the use of hyaluronic acid in the treatment of tendinopathies.

Methods: this review was constructed using the Medline database via Pubmed, Scopus and Google Scholar. The key words hyaluronic acid, tendon and tendinopathy were used for the research.

Results: in total, 28 articles (in English and French) on the application of hyaluronic acid to tendons were selected for their relevance and scientific quality, including 13 for the in vitro part, 7 for the in vivo animal part and 8 for the human section.

Conclusions: preclinical studies demonstrate encouraging results: HA permits tendon gliding, reduces adhesions, creates better tendon architectural organisation and limits inflammation. These laboratory observations appear to be supported by limited but encouraging short-term clinical results on pain and function. However, controlled randomised studies are still needed.

KEY WORDS: hyaluronic acid, tendinopathy, tendon, healing, cicatrization.

\section{Introduction}

Tendon lesions are very common in sportsmen and physical workers ${ }^{1}$. Their physiopathology is multifactorial, including intrinsic and extrinsic factors, which can be modified or not $^{2}$. Even if detrained tendons present the same histological structure as chronic tendinopathies, however, this condition remains an overuse pathology which is often difficult to treat ${ }^{2}$. For this reason, various new treatment have been developed $^{1}$ such as platelet-rich plasma (PRP) ${ }^{3}$ or growth factors ${ }^{4}$ infiltrations. Recently, the anti-inflammatory and lubrication properties of HA have also been suggested for the treatment of these pathologies ${ }^{5}$.

Hyaluronic acid (HA) is a polysaccharide with a high molecular weight (between $100 \mathrm{kDa}$ and $10 \mathrm{MDa}$ ), mainly found in the extracellular matrix where it plays different roles at various places in the organism ${ }^{5}$. Its half-life is only three to five minutes in the circulation, less than a day in tissue, and between one and three weeks in cartilage. This speed of renewal means that this polymer is constantly synthesised and degrad$\mathrm{ed}^{5}$. HA can be categorised into three groups based on molecular weight:

- low molecular weight: $\mathrm{MW}<1 \mathrm{MDa}$;

- medium molecular weight: $1 \mathrm{MDa}<\mathrm{MW}<2 \mathrm{MDa}$;

- $\quad$ high molecular weight: $\mathrm{MW}>2 \mathrm{MDa}$.

$\mathrm{HA}$ ensures the viscoelasticity of liquid connective tissues such as the synovial joint and the corpus vitreum of the eye ${ }^{5}$. As a result of this ability, its role in the body is, among other things, to bind to water and to lubricate mobile parts of the body such as the joints and muscles ${ }^{5}$. Intra-articular injections of synthetic HA are widely used in the medical treatment of osteoarthritis ${ }^{6-8}$.

Industrially, HA is obtained through two different procedures:

- extraction from rooster combs, after grinding and chemical treatment 5 ;

- $\quad$ purification and bacterial fermentation ${ }^{5}$.

The viscosity of the gel which is obtained is proportional to the length (molecular weight, expressed in Daltons) of the polymer fibres. This viscosity will de- 
termine how quickly the product degrades after implantation.

One major absolute contraindication is hypersensitivity to one of the ingredients. Potential undesirable side-effects are pain, a feeling of heat, haematoma, redness and swelling.

\section{Material and methods}

This literature review was constructed using the Medline database via Pubmed, Scopus and Google Scholar, according to the recommendations of the Journal ${ }^{9}$. Articles published in English and French, up to the 30th of September 2015, were found using the following key words alone or in combination: hyaluronic acid, hyaluronan, tendon, tendinopathy, treatment (in English) and acide hyaluronique, tendon, tendinopathie, traitement (in French). In total, only 28 articles on HA as applied to tendons were selected for their relevance and scientific quality: 13 for the in vitro part, 7 for the animal in vivo part and 8 for the human section.

\section{Results}

\section{In vitro experiments}

Even if the first published paper studying the effect of $\mathrm{HA}$ on tendons of rabbits has not shown any effect on the functional strength of tendons after healing in vitro ${ }^{10}$, several Authors have sought to identify the ability of HA to improve tendon gliding.

Sun et al. used 30 canine peroneus longus, randomised into five groups, and immersed for 30 seconds in one of the following five solutions: saline solution, gelatin, carbodiimide and $\mathrm{HA}$ (cd-HA) and gelatin (cd-gelatin) and cd-gelatin- $\mathrm{HA}^{11}$. The tendons were first placed in an incubator at $100 \%$ humidity at $37^{\circ} \mathrm{C}$, before being wrapped in damp cloths for one hour. Gliding resistance for each tendon was measured before the surfaces of the tendons were coated in the various solutions. After 500 cycles, gliding resistance for the tendons coated in saline solution increased significantly, by a factor of 10 , while the cd-gelatin and cd-gelatin-HA did not significantly change. In addition, under microscopy, the surface of the tendons in the saline, gelatin and cd-HA solutions were rough, presenting a network of disorganised fibres. In contrast, the surface of the tendons immersed in the gelatin-cd-HA solution appeared smoother than in the other groups. These observations were confirmed by the study of Nishida et al., which suggest that HA may improve the gliding function of canine flexor tendons ${ }^{12}$.

Taguchi et al. wanted to identify whether these results could be transposed to human tendons ${ }^{13}$. They used 16 human extrasynovial palmaris longus tendons which were divided into two and then immersed randomly in one of the following four solutions: saline solution, carbodiimide-gelatin-HA (cg-gelatin-HA), carbodiimide-gelatin-lubrucin and cd-gelatin-HA-lubrucin. The results, after 1,000 flexion/extension cycles, show that the tendons treated with saline solution presented significantly higher gliding resistance than other groups. These findings, suggesting that HA may limit tendon adhesions, has already been observed in a previous study of Akasaka et al ${ }^{14}$.

In addition to its effects on tendon biomechanics, the researchers looked at the influence of HA on tendon regeneration.

Yamada et al. sought to determine the influence of $\mathrm{HA}$ on cell proliferation and mRNA expression of type I and type III procollagens ${ }^{15}$. They used tendons from 10 subjects suffering from rotator cuff disease who had undergone surgical repair to the cuff. For each fragment, the tendon tissue was separated from the synovial tissue, cut into small pieces and cultured in solutions containing various concentrations of $\mathrm{HA}$ ( 1.0 to $5.0 \mathrm{mg} / \mathrm{mL}$ ). The results showed HA binding to its receptors and a reduction in cell proliferation in a dose-dependent manner in comparison with untreated cells. In addition, the expression level of procollagen a1 (I) mRNA did not significantly reduce in contrast to the expression of procollagen a1 (III) mRNA in a dose-dependent manner.

Another study by Shimpuku et al. analysed the suture site of the supracoracoid tendon in chickens after injection of $\mathrm{HA}^{16}$. As well as confirming that $\mathrm{HA}$ treatment does not increase the production of a1 (I) procollagen, the team was able to show early restoration of tendon continuity at the laceration site in comparison with the control group treated with saline solution. Wiig et al. had compared the effect of HA on cell proliferation and synthesis of matrix component in intrasynovial deep extensor tendons and extrasynovial peroneus tendons of rabbits ${ }^{17}$. They observed that HA modulates cell proliferation unequally in intra- and extrasynovial tendons, but without affecting the matrix component synthesis. This can be partly explained by a difference in biochemical composition and cellular capacities of tendons and by a possible difference in HA sensitivity and in its mechanism of action.

Osti et al. had compared 4 different HA preparation by their molecular weight on human tendon derived cells from rotator cuff tears ${ }^{18}$. They observed that all the HA increased the cell viability and the proliferation at 24 hours compared to control cells (without HA). Furthermore, the synthesis of collagen type I was stimulated over 14 days, without increase in collagen type III. However, these observations were significantly higher in the high molecular weight HA groups.

Neumann et al. looked at the effects of HA on proinflammatory action caused by advanced glycation endproducts (AGEs) ${ }^{19}$. The team compared the efficacy of a preparation of HA with a 'high' molecular weight (HMW-HA - >1.2MDa) to a preparation of $\mathrm{HA}$ with a low molecular weight (LLW-HA - 0.2-0.5MDa) on the production of AGEs and activation of proinflammatory cytokines on murine macrophages. Their results show that HMW-HA, with a molecular weight greater than $1.2 \mathrm{MDa}$, inhibits the inflammation signalling pathway (NF-kB) caused by AGEs and that 
this effect reduces with the molecular weight of $\mathrm{HA}$. Moreover, the results show that low molecular weight $\mathrm{HA}(<0.5 \mathrm{MDa})$ is proinflammatory.

Tanimoto et al. studied the effect of proinflammatory cytokines on HA-synthetase (HAS) mRNA expression using rabbit synovial membrane cells, cultured in inflammatory conditions IL1- $\beta$ and TNF- $\alpha$ were used ${ }^{20}$. Their results showed that proinflammatory cytokines increase HAS mRNA expression and, consequently, may contribute to the accumulation and fragmentation of HA. Low molecular weight HA molecules no longer played their lubricating role in an optimal way. A study by Smith et al. highlighted the effect of adding exongenous HA to endogenous HA synthesis in synovial fibroblasts ${ }^{21}$. Human synovial fibroblasts obtained during biopsies or arthroplasties were cultured. Different molecular weights of HA were then added. The results clearly showed that in vitro HA synthesis by these cells increased when the concentration and molecular weight of the added HA was increased.

The recent study of Nakamura et al., comparing the effects of corticosteroids or HA used in subacromial injection on cell proliferation, in human tendon fibroblasts from rotator cuff tears and in rats after surgical lesion, demonstrated that HA possesses anti-inflammatory and anti-adhesive activities in tendon and synovial fibroblasts. In contrast to CS, HA caused no adverse effects and appears to be relatively safe ${ }^{22}$.

\section{Animal experiments}

Zhao et al. wanted to identify the effect of HA on the reduction of adhesions and on functional improvement after failed tendon reconstruction ${ }^{23}$. To simulate this situation, 28 flexor tendons from the $2^{\text {nd }}$ and $5^{\text {th }}$ digits of dogs were lacerated and repaired. One graft tendon in each dog was treated with an injection of saline solution, and the other with an injection of HA (1\%) combined with lubricin (cd-HA-lubricin). Six weeks after the surgical operation, the allografts treated with cd-HA-lubricin showed decreased adhesions at the proximal extremity, improved digit function and increased graft gliding ability. However, rupture resistance at the proximal and distal repair sites was lower in the treated group than in the control group. Moreover, histological analysis showed delayed healing in the treated group. A study by Oryan et al. aimed to assess the effects of high molecular weight (1.6MDa) HA $(0.9 \%)$ on the healing of flexor tendons in the digits of rabbits after lesion and repair through surgical suture ${ }^{24}$. Injections were administered to 40 rabbits, randomly divided into two equal groups of injured treated with HA and injured untreated. The results showed a significant reduction in the diameter of the treated tendons and an increase in echogenicity. In addition, the dry weight of the injured tendons had significantly increased in comparison with the non-treated tendons. The treatment also significantly improved tenoblast numbers and the diameter and density of collagen fibrils. Yoshida et al. also demonstrated that high molecular weight HA (2700 kDa) was effective for pain relief and for partial restoration of the patellar tendinopathy in a rat model, compared to saline or nothing (control group) ${ }^{25}$.

The Liang et al. team wanted to show the effect of the presence of tenocytes when injecting HA into the Achilles tendons of rats at an early healing stage ${ }^{26}$. After transection and suture of the Achilles tendon, 96 rats were randomised into three groups: one group (THA) received an injection of $1.0 \mathrm{kDa} \mathrm{HA}(1.5 \%)$ with tenocytes $\left(5 \times 10^{5}\right)$, one HA group received the same injection without tenocytes, and a control group received a saline solution injection. A significant difference in recovery, tendon stiffness and locomotive ability was observed in the THA group. The THA group also had a shorter healing time, ending the inflammatory phase and starting the proliferation phase from day 7, while the non-treated and HA groups were still in the inflammatory phase of the healing process on day 7. Acceleration of the inflammatory process through injection of THA may be the main reason for this functional improvement. In addition, the few differences measured between the tendons treated with $\mathrm{HA}$ and the tendons injected with saline solution may be explained by the low molecular weight $(1.0 \mathrm{kDa})$ of HA used. This study shows that adding tenocytes to HA injections may positively influence tendon repair by reducing inflammatory response time. However, the use of low molecular weight HA in this study makes comparisons with THA difficult. Moreover, the use of THA appears to be more appropriate for surgical interventions on tendons than in tendinopathies, given the constraints of removing tenocytes.

The recent series of Salamanna et al. comparing the mechanobiology of patellar tendons of rats randomized into 3 groups (untrained, trained and detrained), demonstrate that discontinuing training activity in the short term alters tenocyte synthetic and metabolic activity and that repeated peri-patellar infiltrations of HA during detraining allow the maintenance of tenocyte anabolic activity 27 : higher proliferation rate and viability, and increased synthesis of C-terminal-propeptide of type I collagen, fibronectin, aggrecan, tenascin-c and matrix-metalloproteinase- 3 . These results are reinforced by those of Frizziero et al., comparing the same groups, who demonstrated that HA is effective in the maintenance of the structural properties of patellar tendon and enthesis in detrained rats ${ }^{28}$.

Contrary of the in vitro observation, Yamaguchi et al. suggested that both hyaluronic acid and steroid injections suppressed of inflammation (evaluated by the expression of calcitonin generelated peptide - CGRP) in a rotator cuff of rats, which thought to be provided pain relief (analyzed by the gait analysis) ${ }^{29}$. While there were no significant differences, the suppression of CGRP expression and the improved gait after hyaluronic acid and steroid injections suggested that both methods were effective for rat rotator cuff tear model.

\section{Human experiments}

Meloni et al. examined the effect of periarticular injection of $\mathrm{HA}$ in patients suffering from a supraspinatus 
tendinopathy ${ }^{30}$. A total of 56 patients participated in the study and were randomised into two equal groups: one group treated with an injection containing $20 \mathrm{mg}$ of $\mathrm{HA}$ (concentration: $20 \mathrm{mg} / 2 \mathrm{ml}$, molecular weight: 0.5-0.7 mDa) in $2 \mathrm{ml}$ of lidocaine (1\%) and $2 \mathrm{ml}$ of physiological liquid $(0.9 \%)$ and a control group receiving an injection of $4 \mathrm{ml}$ of physiological solution $(0.9 \%)$. Lidocaine is used to reduce pain due to the injection. After the first injection, the injection was repeated once a week for four weeks. During this period, analgesics were prescribed for three days after each injection. After one year, 19 of the 28 patients who had received HA were satisfied with their treatment, while all patients in the control group said they were dissatisfied after their injections. Joint extension remained identical throughout the follow-up. At months 1 and 3 following the injection, a significant improvement in pain in the treated group in comparison with the control group was noted. However, after one year, this pain had increased in the treated group. Moreover, no significant difference in tendon structure was observed during the ultrasound scan between the two groups either at the start or at the end of the follow-up.

Petrella et al. treated 330 competitive racquet sports athletes presenting with chronic lateral epicondylosis for more than three months ${ }^{31}$. Two injections were administered one week apart, into the subcutaneous tissue and muscle one centimeter from the lateral epicondyle toward the primary point of pain using a twodimensional fanning technique: the treated group received an injection of $1.2 \mathrm{ml}$ of HA (high but unspecified molecular weight) at $1 \%$ and the control group received a saline solution of $1.2 \mathrm{ml}$. Follow-up examinations were conducted after 7, 14, 30, 90 and 356 days and consisted of a VAS at rest and during measurement of grip strength using a Jamar dynamometer, an elbow disability scale, a functional scale, a satisfaction scale and a measure of time to return to pain-free sport. After one year, there was a significant improvement in pain at rest and grip. Moreover, the scores on all scales had significantly improved in the treated group in comparison with the control group. The time to return to pain-free sport was assessed at 18 days (+/-11 days) in the treated group.

The efficiency of an HA injection for treating patellar tendinopathies was determined in fifty patients ${ }^{32}$ with stage 2 or 3 using Blazina's classification ${ }^{33}$. The injected solution contained $2.5 \mathrm{mg}$ of HA with a molecular weight of $900 \mathrm{kDa}$ in $2.5 \mathrm{ml}$ of physiological liquid and $1 \mathrm{ml}$ of lidocaine. The effectiveness was assessed on the basis of pain during sport, using the modified Roles and Maudsley scores ${ }^{34}$. After treatment, $54 \%$ of cases were 'excellent', and $40 \%$ of cases were 'good'. The results of this study are based on the subjective views of the athletes and their trainers. With a view to reducing post-surgical adhesions of digital flexor tendons, Riccio et al. studied 45 patients randomly assigned to two groups: a control receiving a placebo $(n=10)$, and a treated group receiving an injection of HA (high but unspecified molecular weight) $(n=26)^{35}$. All patients were assessed at 30 ,
60,90 and 180 days after surgery by testing active motion, with functional capacity being assessed using the quick-DASH questionnaire and the number of working days lost after surgery. Patients in the treated group showed better recovery of digital motion at all time intervals (improvement of $27 \%$ in the $\mathrm{HA}$ group compared to $10 \%$ in the control group, and 43 to $28 \%$ respectively at day 180 ), and returned to work earlier. However, no significant difference could be shown in terms of functional score between the two groups.

More recently, HA injections has been used to treat the flexor muscle in the index finger after surgical suture $^{36}$. 11 were treated with three HA injections (0.4 $\mathrm{ml}$ of high molecular weight: 1.0-2.9 MDa and high concentration: $15 \mathrm{mg} / \mathrm{ml}$ ) and 11 received a placebo saline solution of identical volume. Three injections were administered: one at the time of the surgical intervention and two others one week apart. The two groups followed the same rehabilitation protocol after the operation. The results showed no differences between the two groups in terms of extension after three weeks. However, at three months and over the long term, significant functional improvement of total active range of joint motions $(p=0.002$ and $p=0.001$ respectively) was noted in the treated group in comparison with the placebo.

The short- and long-term effect of intra-articular injection of HA in patients suffering from supraspinatus tendinopathy ${ }^{37}$ was assessed in 24 patients randomised into two groups, each received three injections of $2 \mathrm{ml}$ of $\mathrm{HA}(16 \mathrm{mg} / 2 \mathrm{ml}$ with a molecular weight of $6.0 \mathrm{MDa}$ ), following traditional physical therapy treatment. TENS current, ultrasound therapy, thermotherapy (hotpack) and exercises at home (movements, stretches and eccentric exercises). Follow-up consisted of algo-functional score, measurement of active and passive joint extension conducted at three weeks, three months and four years post-injection. A significant improvement in most of the parameters measured was seen within both groups, but no significant difference was observed for the pre-injection VAS in comparison with the VAS conducted at three weeks and three months post-injection.

The series of Merolla et al. enrolled 48 patients with rotator cuff tendinopathy for more than 4 months randomized into 2 groups: one group with 2 ultrasoundguided subacromial injection of HA $(\mathrm{N}=25) 14$ days apart and one group with only a rehabilitation program $(\mathrm{N}=23)^{38}$. The follow-up went up to 6 months. They observed that subacromial HA injections was more effective on pain and algofunctional scores than rehabilitation alone, and this injection was a safe treatment for patients suffering from rotator cuff tendinopathy.

A recent study of Kumai et al. evaluated the effects of a 2,5 mL HA (high-molecular-weight: $2700 \mathrm{kDa}$ ) injections, over a period of 1 week, in a total of 61 patients with enthesopathies: 16 with lateral epicondylitis, 14 with patellar tendinopathy, 15 with insertional Achilles tendinopathy, and 16 with plantar fasciitis ${ }^{39}$. They observed a significant decrease of pain (VAS) for the 4 
sites $(-2.20 \pm 2.26$ for the 4 sites overall), suggesting the efficacy of injections of HA for enthesopathies in the short-term.

\section{Discussion}

Tendinopathies are very common condition in sports and working population ${ }^{1}$. Tendinopathy and tenosynovitis disturbs tendon gliding. The application of HA can reduce tendon glide resistance ${ }^{10-14}$. Several earlier studies have shown the link between the inhibition of fibroblast proliferation and a reduction in type III collagen concentration in the tendon, with a reduction in the formation of adhesions at the tendon healing

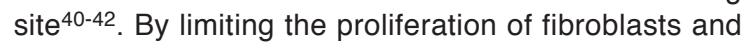
by stabilizing the concentration of type III collagen, $\mathrm{HA}$ may reduce the risk of adhesions ${ }^{10-12}$. It is still discuss if HA can stimulate or not the synthesis of type I collagen to improve the tendon structure and resistance 5,18 , but it is demonstrated that $\mathrm{HA}$ improve cell viability and proliferation ${ }^{17,18}$. HA appears to inhibit the expression of key intermediaries for the inflammatory signaling pathways (NF-kB), in a dosedependent manner ${ }^{19,43}$. By reducing the expression of proinflammatory factors, adding exogenous $\mathrm{HA}$ reduces the fragmentation of endogenous HA and stimulates synovial synthesis of endogenous $\mathrm{HA}^{16,26,36}$. Concerning the therapeutic protocol for HA injections for tendinopathies, comparatively to other recent treatments for these conditions, where post-injection rehabilitation could be crucial ${ }^{44}$, such as PRP for example ${ }^{45}$, only 1 or 2 injections alone are described for HA treatments $30-32,35-39$. However, comparatively to PRP treatments, where it exist a wide variability in the administered product ${ }^{46,47}$, the molecular weight and the concentration of $\mathrm{HA}$ are known ${ }^{18}$; high molecular weight HA seems to have a better effect on tendons $^{18}$ in the short-term.

\section{Conclusion}

Some in vitro and animal in vivo studies demonstrate encouraging results in terms of the ability of $\mathrm{HA}$ to encourage tendon gliding and to reduce adhesions as well as creating better tendon architectural organisation. Moreover, the injection of HA may limit the proinflammatory effect by restoring viscoelasticity and by stimulating the endogenous synthesis of $\mathrm{HA}$. These laboratory observations appear to be supported by limited but encouraging short-term clinical results on pain and function as well as on the return to sport. However, controlled randomised studies are still needed.

\section{References}

1. Kaux JF, Forthomme B, Le Goff C, Crielaard JM, Croisier JL. Current Opinions on Tendinopathy. J Sports Sci Med. 2011; 10:238-253.
2. Kaux JF, Crielaard JM. Tendon et tendinopathie. J Traumatol Sport. 2014;31:235-240.

3. Kaux JF, Drion P, Croisier JL, Crielaard JM. Tendinopathies and platelet-rich plasma (PRP): from pre-clinical experiments to therapeutic use. J Stem Cells Regen Med. 2015;11:7-17.

4. Kaux JF, Janssen L, Drion P, et al. Vascular Endothelial Growth Factor-111 (VEGF-111) and tendon healing: preliminary results in a rat model of tendon injury. Muscles Ligaments Tendons J. 2014;8:24-28.

5. Necas J, Bartosikova L, Brauner P, Kolar J. Hyaluronic acid (hyaluronan): a review, Veterinarni Medicina. 2008;53:397-411.

6. Jordan KM, Arden NK, Doherty M, et al. EULAR Recommendations 2003: an evidence based approach to the management of knee osteoarthritis: Report of a Task Force of the Standing Committee for International Clinical Studies Including Therapeutic Trials (ESCISIT). Ann Rheum Dis. 2003;62:1145-1155.

7. Zhang W, Doherty M, Arden N, et al. EULAR evidence based recommendations for the management of hip osteoarthritis: report of a task force of the EULAR Standing Committee for International Clinical Studies Including Therapeutics (ESCISIT). Ann Rheum Dis. 2005;64:669-681.

8. Zhang W, Moskowitz RW, Nuki G, et al. OARSI recommendations for the management of hip and knee osteoarthritis, part I: critical appraisal of existing treatment guidelines and systematic review of current research evidence. Osteoarthritis Cartilage. 2007;15:981-1000.

9. Padulo S, Oliva F, Frizziero A, Maffulli N. Muscles, Ligaments and Tendons Journal. Basic principles and recommendations in clinical and field science research. MLTJ. 2013;4:250-252.

10. Salti NI, Tuel RJ, Mass DP. Effect of hyaluronic acid on rabbit profundus flexor tendon healing in vitro. J Surg Res. 1993; 55:411-415.

11. Sun YL, Yang C, Amadio PC, Zhao C, Zobitz ME, An KN. Reducing friction by chemically modifying the surface of extrasynovial tendon grafts. J Orthop Res. 2004;22:984-989.

12. Nishida J, Araki S, Akasaka T, Toba T, Shimamura T, Amadio $\mathrm{PC}, \mathrm{An} \mathrm{KN}$. Effect of hyaluronic acid on the excursion resistance of tendon grafts. A biomechanical study in a canine model in vitro. J Bone Joint Surg Br. 2004;86:918-924.

13. Taguchi M, Zhao C, Sun YL, Jay GD, An KN, Amadio PC. The Effect of Surface Treatment Using Hyaluronic Acid and Lubricin on the Gliding Resistance of Human Extrasynovial Tendons In Vitro. J Hand Surg Am. 2009;34:1276-1281.

14. Akasaka T, Nishida J, Imaeda T, Shimamura T, Amadio PC, An KN. Effect of hyaluronic acid on the excursion resistance of tendon graft: a biomechanical in vitro study in a modified human model. Clin Biomech (Bristol, Avon). 2006;21:810-815.

15. Yamada T, Gotoh M, Nakama K, Mitsui Y, Higuchi F, Nagata $\mathrm{K}$. Effects of hyaluronan on cell proliferation and mRNA expression of procollagens alpha 1 (I) and alpha 1 (III) in tendonderived fibroblasts from patients with rotator cuff disease: an in vitro study. Am J Sports Med. 2007;35:1870-1876.

16. Shimpuku E, Hamada K, Handa A, et al. Molecular effects of sodium hyaluronate on the healing of avian supracoracoid tendon tear: according to in situ hybridization and real-time polymerase chain reaction. J Orthop Res. 2007;25:173-184.

17. Wiig M, Abrahamsson SO. Hyaluronic acid modulates cell proliferation unequally in intrasynovial and extrasynovial rabbit tendons in vitro. J Hand Surg Br. 2000;25:183-187.

18. Osti L, Berardocco M, di Giacomo V, Di Bernardo G, Oliva F, Berardi AC. Hyaluronic acid increases tendon derived cell viability and collagen type I expression in vitro: Comparative study of four different Hyaluronic acid preparations by molecular weight. BMC Musculoskelet Disord. 2015;16(1):284.

19. Neumann A, Schinzel R, Palm D, Riederer P, Münch G. High molecular weight hyaluronic acid inhibits advanced glycation endproduct-induced NF-kB activation and cytokine expression. Febs Letters. 1999;453:283-287. 
20. Tanimoto K, Ohno S, Fujimoto K, et al. Proinflammatory cytokines regulate the gene expression of hyaluronic acid synthetase in cultured rabbit synovial membrane cells. Connect Tissue Res. 2001;42:187-195.

21. Smith MM, Ghosh P. The synthesis of hyaluronic acid by human synovial fibroblasts is influenced by the nature of the hyaluronate in the extracellular environment. Rheumatol Int. 1987;7:113-122.

22. Nakamura H, Gotoh M, Kanazawa T, et al. Effects of corticosteroids and hyaluronic acid on torn rotator cuff tendons in vitro and in rats. J Orthop Res. 2015;33:1523-1530.

23. Zhao C, Wei Z, Kirk RL, et al. The Effects of Bio-Lubricating Molecules on Flexor Tendon Reconstruction in A Canine Allograft Model In Vivo. Plast Reconstr Surg. 2014;133:628e637e.

24. Oryan A, Moshiri A, Meimandi Parizi AH, Raayat Jahromi A. Repeated administration of exogenous Sodium-hyaluronate improved tendon healing in an in vivo transection model. J Tissue Viability. 2012;21:88-102.

25. Yoshida M, Funasaki H, Kubota M, Marumo K. Therapeutic effects of high molecular weight hyaluronan injections for tendinopathy in a rat model. J Orthop Sci. 2015;20(1):186-195.

26. Liang JI, Lin PC, Chen MY, Hsieh TH, Chen JJ, Yeh ML. The effect of tenocyte/hyaluronic acid therapy on the early recovery of healing Achilles tendon in rats. J Mater Sci Mater Med. 2014;25:217-227.

27. Salamanna F, Frizziero A, Pagani S, et al. Metabolic and cytoprotective effects of in vivo peri-patellar hyaluronic acid injections in cultured tenocytes. Connect Tissue Res. 2015;56:35-43.

28. Frizziero A, Salamanna F, Giavaresi G, et al. Hyaluronic acid injections protect patellar tendon from detraining-associated damage. Histol Histopathol. 2015;30:1079-1088.

29. Yamaguchi T, Ochiai N, Sasaki $Y$, et al. Efficacy of hyaluronic acid or steroid injections for the treatment of a rat model of rotator cuff injury. J Orthop Res. 2015.

30. Meloni F, Milia F, Cavazzuti M, et al. Clinical evaluation of sodium hyaluronate in the treatment of patients with sopraspinatus tendinosis under echographic guide: Experimental study of periarticular injections. Eur J Radiol. 2008;68:170-173.

31. Petrella RJ, Cogliano A, Decaria J, Mohamed N, Lee R. Management of Tennis Elbow with sodium hyaluronate periarticular injections. Sports Med Arthrosc Rehabil Ther Technol. 2010;2;2-4.

32. Muneta T, Koga H, Ju YJ, Mochizuki T, Sekiya I. Hyaluronan injection therapy for athletic patients with patellar tendinopathy. J Orthop Sci. 2012;17:425-431.

33. Blazina ME, Kerlan RK, Jobe FW, Carter VS, Carlson GJ. Jumper's knee. Orthop Clin North Am. 1973;4:665-678.

34. Roles NC, Maudsley RH. Radial tunnel syndrome: resistant tennis elbow as a nerve entrapment. J Bone Joint Surg Br. 1972;54(3):499-508.

35. Riccio M, Battiston B, Pajardi G, et al. Efficiency of Hyaloglide in the prevention of the recurrence of adhesions after tenolysis of flexor tendons in zone II: a randomized, controlled, multicentre clinical trial. J Hand Surg Eur. 2010;35:130-138.

36. Özgenel GY, Etöz A. Effects of repetitive injections of hyaluronic acid on peritendinous adhesions after flexor tendon repair: a preliminary randomized, placebo-controlled clinical trial. Ulus Travma Acil Cerrahi Derg. 2012;18:11-17.

37. Ozgen M, Fırat S, Sarsan A, Topuz O, Ardı̧ F, Baydemir C Short- and long-term results of clinical effectiveness of sodium hyaluronate injection in supraspinatus tendinitis. Rheumatol Int. 2012;32:137-144.

38. Merolla G, Bianchi P, Porcellini G. Ultrasound-guided subacromial injections of sodium hyaluronate for the management of rotator cuff tendinopathy: a prospective comparative study with rehabilitation therapy. Musculoskelet Surg. 2013;97 Suppl 1:49-56.

39. Kumai T, Muneta T, Tsuchiya A, et al. The short-term effect after a single injection of high-molecular-weight hyaluronic acid in patients with enthesopathies (lateral epicondylitis, patellar tendinopathy, insertional Achilles tendinopathy, and plantar fasciitis): a preliminary study. J Orthop Sci. 2014;19(4):603-611.

40. Al-Qattan MM. Factors in the pathogenesis of Dupuytren's contracture. J Hand Surg [Am]. 2006;31:1527-1534.

41. Tomonaga A, Hamada K, Gotoh M, Yamakawa H, Kobayashi $\mathrm{K}$, Fukuda $\mathrm{H}$. Expression of procollagen alpha 1 type III mRNA in rotator cuff tears. Tokai J Exp Clin Med. 2000;25(3):125-134.

42. Wiig M, Abrahamsson SO, Lundborg G. Effects of hyaluronan on cell proliferation and collagen synthesis: a study of rabbit flexor tendons in vitro. J Hand Surg Am. 1997;22:818-825.

43. Moreland LW. Intra-articular hyaluronan (hyaluronic acid) and hylans for the treatment of osteoarthritis: mechanisms of action. Arthritis Res Ther. 2003;5:54-67.

44. Kaux JF, Forthomme B, Namurois MH, et al. Description of a standardized rehabilitation program based on sub-maximal eccentric following a platelet-rich plasma infiltration for jumper's knee. Muscles Ligaments Tendons J. 2014;4:85-89.

45. Kaux JF, Bouvard M, Lecut C, et al. Reflections about the optimisation of the treatment of tendinopathies with PRP. Muscles Ligaments Tendons J. 2015;5:1-4.

46. Kaux JF, Le Goff C, Renouf J, et al. Comparison of the platelet concentrations obtained in platelet-rich plasma (PRP) between the GPS ${ }^{\mathrm{TM}}$ II and GPS ${ }^{\mathrm{TM}}$ III systems. Pathol Biol (Paris). 2011;59:275-277.

47. Kaux JF, Le Goff C, Seidel L, et al. Comparative study of five techniques of preparation of platelet-rich plasma. Pathol Biol (Paris). 2011;59:157-160. 